\title{
РЕГУЛИРОВАНИЕ ВОСПРОИЗВОДИМОСТИ ПАРАМЕТРОВ ПОЛИМЕРНЫХ РЕЗИСТОРОВ ПРИ ПОВЫШЕННЫХ ТЕМПЕРАТУРАХ
}

\author{
Минакова Наталья Николаевна 1 , \\ minakova@asu.ru \\ Ушаков Василий Яковлевич², \\ vyush@tpu.ru \\ 1 Алтайский государственный университет, \\ Россия, 656049, Барнаул, пр. Ленина, 61. \\ 2 Национальный исследовательский Томский политехнический университет, \\ Россия, 634050, г. Томск, пр. Ленина, 30.
}

\begin{abstract}
Актуальность работы обусловлена тем, что резистивные полимерные материалы активно применяются в различных электротехнических устройствах. Некоторые из них изготавливаются из наполненных техническим углеродом каучуков по хорошо освоенной промышленной технологии из доступного сырья. Дальнейшее увеличение масштабов их применения сдерживает сложность выбора компонентов для обеспечения требуемых электросизических свойств. Разброс параметров, связанный с многокомпонентностью структуры, увеличивает стоимость и сырья для изготовления готовых изделий, и технологии.

Цель: оценка возможности улучшения воспроизводимости свойств в серии электротехнических изделий для конкретных условий эксплуатации за счет рецептурных приемов. Проблема усугубляется многокомпонентностью структуры, которая затрудняет обеспечение стабильности и воспроизводимости электросизических свойств. Для обеспечения возможности использования результатов в промышленных технологиях электротехнические изделия и образцы изготавливались из композиционных материалов, которые отличались концентрацией, маркой электропроводящего компонента, видом полимера.

Методы: инструментальные измерения электрофизических характеристик резистивных полимерных композиционных материалов, дисперсионный, регрессионный и корреляционный анализы.

Результаты. Определены закономерности изменения коэфффициента вариации и величины температурного коэфффициента сопротивления от концентрации электропроводящего наполнителя (технического углерода), вида каучука при нагреве постоянным и переменным током в диапазоне приложенного напряжения до 1000 В. Разработаны рекомендации по повышению однородности структуры полимерных композиционных материалов в условиях их эксплуатации при повышенных температурах. Их применение способствует сокращению используемых ресурсов (энергии, материалов, времени) благодаря повышению воспроизводимости величины объемного электрического сопротивления при нагреве в процессе эксплуатации электротехнических изделий.
\end{abstract}

\section{Ключевые слова:}

Ресурсосбережение, наполненные полимеры, объемное электрическое сопротивление, температурный коэффрициент сопротивления, коэффициент вариации, повышенная температура.

\section{Введение}

Резистивные полимерные композиционные материалы применяются для изготовления широкой гаммы электротехнических изделий: резисторов, нагревателей, экранов кабелей, антистатических устройств и др. [1-3]. Для расширения масштабов их применения и улучшения потребительских свойств необходимо создать материалы с заданной величиной объемного электрического сопротивления (ОЭС), что достигается оптимальным подбором вида полимера, марки и концентрации электропроводящего наполнителя. Одновременно требуется решение задачи повышения эффективности использования материалов и изделий из них.

В наполненных полимерах протекает комплекс сложных, трудно контролируемых процессов, приводящих к формированию межфазной поверхности соприкосновения проводящего наполнителя с полимером [4-8]. Из-за многокомпонентности структуры и сложности технологии изготовления, включающей однородное распределение электропроводящего наполнителя в полимерной матрице, величина ОЭС не может быть рассчитана аналитически и всегда имеет определенной разброс [9, 10]. При его существенной величине затраченные материальные, временные и другие ресурсы используются неэффективно. Для уменьшения такого разброса требуется изучение влияния основных компонентов резистивного полимерного композиционного материала на воспроизводимость эксплуатационных параметров при серийном производстве.

В научных публикациях можно встретить отрывочные сведения о результатах экспериментального определения воспроизводимости свойств резистивных полимерных композиционных материалов. Обычно изучается величина ОЭС в серии изделий после технологического цикла. Экспериментально подтверждено, что воспроизводимость величины ОЭС при серийном производстве снижается при уменьшении концентрации электропроводящего наполнителя из-за сложности его однородного распределения по объёму матрицы $[11,12]$.

Оценка воспроизводимости величины ОЭС в серии изделий после технологического цикла не позво- 
ляет в полной мере оценить эффективность использования ресурсов при заданных требованиях к надежности эксплуатации электротехнических устройств.

Главный эксплуатационный параметр материалов электротехнических устройств в слабых электрических полях - температура. Она определяющим образом влияет на величину ОЭС. Воздействие температуры может существенно повлиять на величину воспроизводимости эксплуатационных параметров в серии, снижая работоспособность электротехнического устройства из-за ухудшения качества материала.

Сведения об исследовании воспроизводимости резистивных полимерных композиционных материалов при нагреве в литературе не обнаружены. Представляло интерес выявить связь разброса электрофизических параметров в серии изделий при действии температуры со свойствами основных компонентов материала.

\section{Объекты и методы исследования}

В качестве объекта исследования выбраны наполненные техническим углеродом каучуки, которые характеризуются доступностью исходных компонентов и высоким уровнем отработанности технологии изготовления $[13,14]$. Изучались материалы, отличающиеся видом каучука, концентрацией и маркой технического углерода промышленных марок. Электропроводящий наполнитель - технический углерод различной структурности: от низкоструктурного (П-514) до высокоструктурного электропроводящего (П-366 Э) Концентрация определена как отношение массы электропроводящего наполнителя к массе материала матрицы (массовые части наполнителя на 100 массовых частей каучука). Проанализированы материалы с концентрацией технического углерода от 30 до 80 массовых частей на 100 массовых частей каучука.

Как известно, кристаллические полимеры имеют дальний порядок расположения молекул, а аморфные полимеры - лишь ближний порядок. Структурная организация аморфных полимеров - наличие упорядоченных областей, чередующихся с областями беспорядка, малые размеры упорядоченных участков [5]. Такая полидисперсность приводит к разбросу показателей. В этой связи эксперименты выполнялись с материалами, имеющими в качестве матрицы кристаллический каучук (Butil Rubber, IIR по ASTM), в РФ - БК2055 , и аморфный бутадиен-метилстирольный каучук Styrene Butadiene Rubber (SBR по ASTM), в РФ CКМС-30АРК. В выбранных объектах исследования материалы матрицы различаются химическим строением, морфологией их надмолекулярной структуры, видом и интенсивностью межмолекулярных связей.

Технология изготовления резистивных полимерных композиционных материалов включала стандартные для шинной и резинотехнической промышленности этапы: смешение, профилирование изделия, вулканизация $[10,11]$. Дополнительно выполнялось разделение заготовки на изделия необходимых размеров, нанесение электродов на изделия. Образцы для испытаний вулканизировались в виде цилиндров диаметром 0,03 м, высотой 0,05 м.
Измерялась величина ОЭС объектов исследования после технологического цикла и до начала испытаний $\left(\rho_{v}\right)$, после приложения постоянного и переменного напряжения различной величины и длительности $\left(\rho_{v}\right)$, температурный коэффициент сопротивления (ТКС), температура нагрева $t$. Измерения $\rho_{v}$ и ТКС выполнялись по стандартным методикам. Диапазон температур при определении температурного коэффициента сопротивления составлял $+20-+200{ }^{\circ} \mathrm{C}$ (ГОСТ 21342.15-78 с изменениями, скорость нагрева $3-5{ }^{\circ} \mathrm{C} /$ мин).

Температура измерялась в центре образцарезистора с помощью термопары общепромышленного назначения (хромель-копелевая термопара, отградуированная в соответствии с требованиями ГОСТ). Термопары были внедрены в тело резистора и изолированы слоем эпоксидной смолы или фторопластовой трубкой.

Характеристикой однородности структуры служил коэффициент вариации равномерности распределения фазовых составляющих $K(\%)$, величина которого характеризует степень отклонения распределения анализируемой фазы от однородного распределения (при уменьшении величины коэффициента вариации повышается однородность структуры). Сравнение рассеяния случайных значений параметров выполнялось по величине коэффициента вариации $K, \%$, в выборке, содержащей не менее 25 изделий. Коэффициент вариации рассчитывался как отношение среднеквадратичного отклонения значения параметра в выборке к его среднему значению.

Сравнивались коэффициенты вариации значений температурного коэффициента сопротивления при изменении концентрации электропроводящего наполнителя в диапазоне 30-80 мас. \% (массовых частей на 100 массовых частей матрицы) при разных материалах матрицы.

При обработке результатов измерений использованы стандартные статистические и математические пакеты, включающие методы описательной статистики, дисперсионного и регрессионного анализа. С их помощью выполнялся расчет полиномиальных уравнений регрессии, оценка их значимости, подсчет функций отклика, оценка значимости влияющих факторов и т. д. Статистически значимые отличия при сравнении выборок определялись с помощью T-test (проводился с уровнем значимости 0,05 ).

\section{Результаты исследования}

Результаты экспериментальных исследований представлены на рис. 1-3 и в таблице.

На рис. 1 в качестве примера представлен график зависимости коэффициента вариации величины ТКС в партии изделий от концентрации электропроводящего наполнителя.

Установлено, что данная закономерность примерно одинакова для матриц обеих марок. По виду она сходна с закономерностью изменения коэффициента вариации величин $\rho_{v}$ после технологического цикла при серийном изготовлении - при увеличении концентрации наполнителя коэффициент вариации уменьшается. 


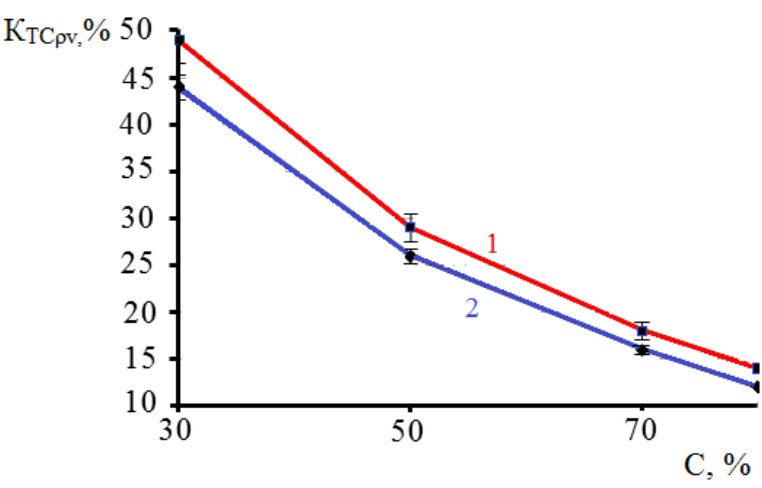

Рис. 1. Зависимость коэффициента вариации величины ТКС от концентрации наполнителя: 1 - матрииа СКМС-30АРК, наполнитель П-234; 2 - матрица БК-2055, наполнитель П-234

Fig. 1. Dependence of the temperature coefficient of resistance (TCR) value variation coefficient on concentration of the filler: 1 - matrix SKMS-30ARK, filler $P$-234; 2 - matrix $B K-2055$, filler $P$-234

На резисторы-образцы подавалось напряжение различной величины и длительности. Оценивался разброс $\rho_{v}$ при нагреве $\left(\rho_{v}\right)$. На рис. 2 представлены результаты расчета коэффициента вариации величины $\rho_{v}$ при изменении воздействующего напряжения в диапазоне 250-1000 В.

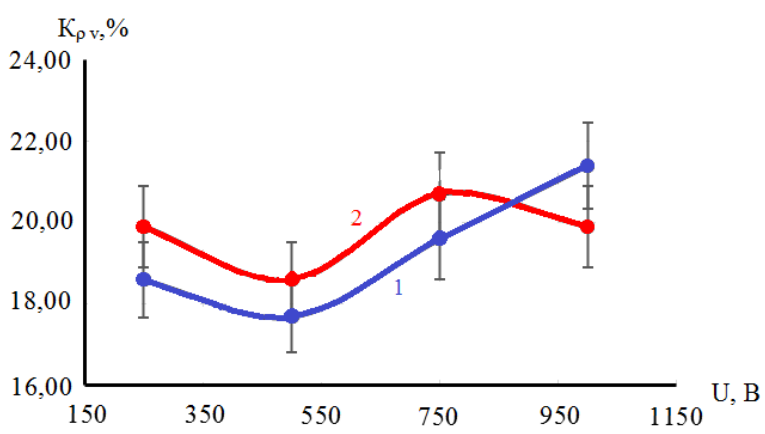

Pис. 2. Зависимость коэффичиента вариачии величинь объемного электрического сопротивления при нагреве от величины приложенного напряжения: 1 - переменное, 2 - постоянное (материал: матрица БК-2055, электропроводящий наполнитель - П-234, концентрация - 60 мас. \%)

Fig. 2. Dependence of the coefficient of variation of the volumetric electrical resistance during heating on the value of the applied voltage: 1 -variable, $2-$ constant (material: matrix BK-2055, electrically conductive filler $-P-234$, concentration $-60 \mathrm{wt} . \%)$

Анализ результатов экспериментов показал, что графики зависимости изменения коэффициента вариации от характера приложенного напряжения (переменный, постоянный) практически однотипны, с учетом пятипроцентного статистического разброса параметров. Род тока в проведенных экспериментах не влияет на коэффициент вариации $\rho_{v}$ в партии образцов одинакового состава.

Представляло интерес сопоставить результаты расчета коэффициента вариации для $\rho_{v}$, ТКС, величи- ны температуры в теле резистора (серия включала 25 изделий).

Известно, что полимер более подвержен действию высоких температур, чем электропроводящий наполнитель $[12,13]$. По этой причине эксперименты проводились на материалах, содержащих разные матрицы. В таблице результаты экспериментов представлены для материалов, содержащих в качестве матрицы каучуки марок СКМС-30АРК, БК-2055. Электропроводящий наполнитель - технический углерод промышленных марок П-514, П-234, П-166Э при концентрации 80 массовых частей на 100 массовых частей каучука (разброс параметров в серии не превышал 5 \% от указанных в таблице средних значений).

Таблица. Основные результаты экспериментов (измерений)

Table. $\quad$ The main results of the experiments (measurements)

\begin{tabular}{|c|c|c|c|c|c|c|}
\hline \multirow{4}{*}{ 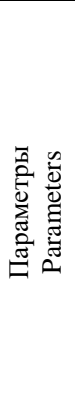 } & \multicolumn{6}{|c|}{$\begin{array}{l}\text { Электропроводящий наполнитель } \\
\text { Electrically conductive filler }\end{array}$} \\
\hline & \multicolumn{2}{|c|}{$\begin{array}{l}\Pi-166 Э \\
\text { Р-166E }\end{array}$} & \multicolumn{2}{|c|}{$\begin{array}{l}\text { П-234 } \\
\mathrm{P}-234\end{array}$} & \multicolumn{2}{|c|}{$\begin{array}{r}\Pi-514 \\
\text { Р-514 }\end{array}$} \\
\hline & \multicolumn{6}{|c|}{$\begin{array}{c}\text { Материал матрицы } \\
\text { Matrix material }\end{array}$} \\
\hline & 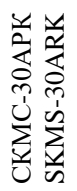 & 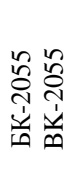 & 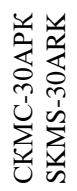 & 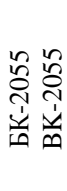 & 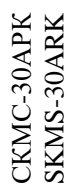 & 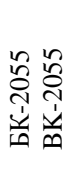 \\
\hline $\mathrm{K}_{\mathrm{\rho v}}$ & 8 & 11,8 & 10,3 & 14,4 & 16 & 18,4 \\
\hline $\begin{array}{l}\mathrm{K}_{\mathrm{TK} \rho \mathrm{v}} \\
\mathrm{K}_{\mathrm{TC} \rho \mathrm{v}}\end{array}$ & 13,9 & 12,1 & 16,9 & 14,9 & 20,8 & 19,4 \\
\hline $\mathrm{K}_{\mathrm{t}}$ & 10 & 8 & 12 & 9 & 15 & 13 \\
\hline
\end{tabular}

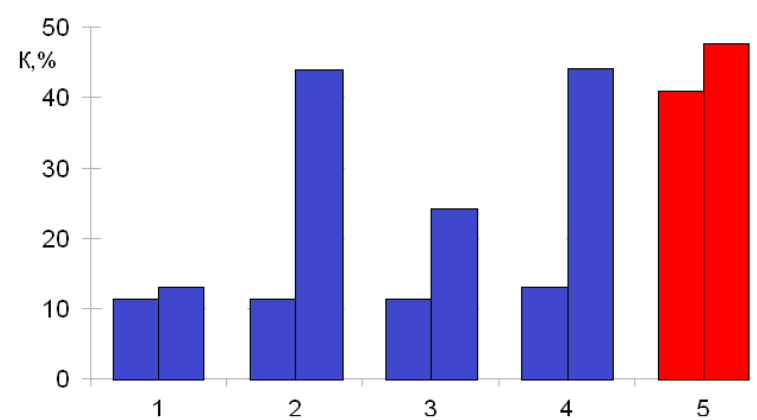

Pис. 3. Зависимость коэффицуиента вариациии $\rho_{v}$ от величины напряжения и длительности его воздействия: 1-4-низкоомный материал, 5-высокоомный материал. Левые столбиь: 1 - величина напряжения $60 \mathrm{~B}$, длительность $72 \mathrm{c} ; 2-600 \mathrm{~B}$, $72 c ; 3-60 \mathrm{~B}, 72 c ; 4-60 \mathrm{~B}, 720 c ; 5-60 \mathrm{~B}, 72 c$; правые столбиы: $1-60 \mathrm{~B}, 720 \mathrm{c} ; 2-600 \mathrm{~B}, 720 \mathrm{c}$; $3-600$ B, $72 c ; 4-600$ B, $720 c ; 5-600$ B, $720 c$

Fig. 3. Dependence of the coefficient of variation $\rho_{v}$ on voltage magnitude and duration of its exposure: 1-4 - low resistance material, 5 - high resistance material. Left columns: 1 - voltage magnitude $60 \mathrm{~V}$, duration $72 \mathrm{~s} ; 2-600 \mathrm{~V}, 72 \mathrm{~s} ; 3-60 \mathrm{~V}, 72 \mathrm{~s} ; 4-$ $60 \mathrm{~V}, 720 \mathrm{~s} ; 5-60 \mathrm{~V}, 72 \mathrm{~s}$; right columns: $1-60 \mathrm{~V}$, $720 \mathrm{~s} ; 2-600 \mathrm{~V}, 720 \mathrm{~s} ; 3-600 \mathrm{~V}, 72 \mathrm{~s} ; 4-600 \mathrm{~V}$, $720 \mathrm{~s} ; 5-600 \mathrm{~V}, 720 \mathrm{~s}$ 
Результаты экспериментов показали, что коэффициент вариации величин ТКС, температуры и $\rho_{v}$ при нагреве зависит от вида полимера и наполнителя. Коэффициент вариации $\rho_{v}$ резистора до испытаний при замене материала матрицы с аморфного на кристаллический увеличивается, при нагреве закономерность обратная - величина коэффициента уменьшается или сохраняется практически неизменной. Закономерности изменения коэффициента вариации в серии значений ТКС и температуры совпадают.

На рис. 3 представлены зависимости коэффициента вариации $\rho_{v}$ от величины и длительности воздействия напряжения для материалов, отличающихся значениями $\rho_{v}$ более чем на порядок (высокоомный 540,7 Ом м, низкоомный - 3,2 Ом м).

Анализ результатов, представленных на рис. 3, позволил выявить следующие закономерности.

1. При увеличении напряжения величина $K$ (разброс параметра ОЭС в партии образцов одинакового состава из-за многокомпонентности структуры материала, связанный с неоднородным распределением технического углерода в каучуке) возрастает как для высокоомных, так и для низкоомных материалов.

2. Увеличение длительности воздействия напряжения малой величины (60 В) на низкоомные материалы практически не влияет на коэффициент вариации величины $\rho_{v t}$. При возрастании ОЭС влияние времени приложения напряжения на коэффициент вариации возрастает даже при малой величине напряжения. Различие в величинах $K$ для высокоомного и низкоомного материалов в проведенных экспериментах превышает двукратное).

3. Изменение длительности воздействия напряжения влияет на величину $K$ сильнее, чем изменение его величины. Этот эффект усиливается в области высоких значений напряжения.

Значения коэффициентов вариации $\rho_{v}$ при серийном изготовлении для различных уровней температуры сравнивались по законам распределения (T-test проводился с уровнем значимости 0,05). Установлено, что законы распределения случайных значений $\rho_{v t}$ и $t$ совпадают. Законы распределения выборок (25 объектов) коэффициента вариации $\rho_{v t}$ в партии изделий после технологического цикла и при нагреве отличаются. Из-за выявленного различия в законах распределения представляло интерес определить степень значимости по вкладу основных рецептурных компонентов в формирование коэффициента вариации изучаемых параметров. Установлено, что порядок влияния основных компонентов на коэффициент вариации различен. Степень значимости основных рецептурных компонентов по влиянию на разброс величины сопротивления после технологического цикла имеет следующую последовательность - концентрация, марка технического углерода, вид полимера; разброс по изменению $\rho_{v t}$ при нагреве имеет другую последовательность влияния факторов - концентрация, вид полимера, марка технического углерода.

Воздействие повышенных температур на полимерные композиционные материалы приводит к ком- плексу разнообразных процессов: термоокислительная деструкция, механические деформации, изменяющие зазор в проводящих контактах, релаксация механических напряжений и химических процессов, структурирование и т. д. [4, 5, 13-16]. Результаты экспериментов по разбросу исходного объемного электрического сопротивления и при нагреве можно связать с разной степенью влияния на процессы электропроводности и тепловые процессы ряда факторов, например, плотности полимеров $[5,8]$. Теплопроводность полимеров в значительной степени связывают с их плотностью: при повышении степени кристалличности полимера его теплопроводность выше. Степень влияния плотности полимера на электропроводность композиционного материала является менее существенной $[8,13]$. В ряде работ [17-21] большое внимание уделено изучению влияния различных факторов (прежде всего, температуры) не только на электропроводность наполненных полимеров, но и на другие их физические свойства: термомеханические, барьерные и др.

\section{Заключение}

Производство и использование электротехнических изделий из полимерных композиционных материалов сдерживаются сложностью выбора компонентов, обеспечивающих требуемые потребительские свойства. Опыт производства и эксплуатации электротехнических изделий из композиционных материалов показывает, что на их конкурентоспособность по показателям «цена - потребительские качества» существенно влияет воспроизводимость и стабильность рабочих характеристик, которые, в свою очередь, определяются поведением материалов в процессе переработки их в изделия.

Комплекс выполненных исследований включал:

- изучение на серии изделий влияния на воспроизводимость величины $\rho v$ и ТКС наполненных техническим углеродом каучуков следующих факторов: температуры, величины $\rho v$, величины и длительности воздействия электрического поля, рода тока (постоянный, переменный);

- анализ зависимости коэффициента вариации значений $\rho v$ при действии повышенных температур от основных рецептурных параметров (концентрации и марки технического углерод, вида полимера).

Установлено, что зависимость коэффициента вариации и величины ТКС от концентрации подчиняется следующей закономерности: при уменьшении концентрации разброс возрастает. Закономерность справедлива для всех используемых в исследованиях марок электропроводящего наполнителя и видов полимера.

Результаты экспериментов подтвердили, что коэффициент вариации объемного электрического сопротивления при нагреве постоянным и переменным током в исследуемом диапазоне приложенного напряжения до 1000 В практически не изменяется по величине. 
Выявлена возможность направленного регулирования коэффициента вариации $\rho_{v}$ полимерных резисторов при нагреве в слабых электрических полях. Для уменьшения разброса сопротивления в серии изделий в результате повышения температуры необходима корректировка состава материала - регулировать требуемую электропроводность в условиях высокой концентрации наполнителя, используя для этого стойкий к действию температуры полимер. Замена низкоструктурного технического углерода на высокоструктурный, принципиально важная для формирования величины $\rho_{v}$ после технологического цикла,

\section{СПИСОК ЛИТЕРАТУРЫ}

1. Stress softening of carbon black filled SBRs submitted to various large strain uniaxial tension cycles / M. Brieu, J. Diani, C. Mignot, C. Moriceau // Procedia Engineering. - 2010. - № 1 (2). P. 1291-1296.

2. Polymer/carbon based composites as electromagnetic interference (EMI) shielding materials / J.-M. Thomassina, C. Jérômea, T. Pardoenb, C. Baillyb, I. Huynenb, C. Detrembleura // Materials Science and Engineering. - 2013. - V. 74. - Iss. 7. - P. 211-232.

3. Zhang W., Dehghani-Sanij A.A., Blackburn. R.S. Carbon based conductive polymer composites // Journal Materials Science. 2007. - V. 42. - P. 3408-3418.

4. Аскадский А.А., Хохлов А.Р. Введение в физико-химию полимеров. - М.: Научный мир, 2009. - 384 с.

5. Липатов Ю.С. Физико-химические основы наполнения полимеров. - М.: Химия, 1977. $-260 \mathrm{c}$.

6. Electrical conduction hysteresis in carbon black-filled butyl rubber compounds / M.A. Alzamil, K. Alfaramawi, S. Abboudy, L. Abulnasr // International Journal of Modern Physics. - 2018. № 32 (09). - P. 1-10.

7. Berki P., Göbl R., Karger-Kocsis J. Structure and properties of styrene-butadiene rubber (SBR) with pyrolytic and industrial carbon black // Polymer Testing. - 2017. - V. 61. - P. 404-415.

8. Szadkowski B., Marzec A., Zaborski M. Effect of different carbon fillers on the properties of nitrile rubber composites // Composite Interfaces. - 2019. - V. 26 (8). - P. 729-750.

9. Janke W. Computer simulation studies of polymer adsorption and aggregation - from flexible to stiff // Physics Procedia. - 2015. № 68. - P. 69-79.

10. The role of conductive pathways in the conductivity and rheological behavior of poly (methyl methacrylate)-graphite composites / X. Liu, Y. Pan, X. Hao, K. Dai, D.W. Schubert / Journal of Applied Polymer Science. - 2016. - № 133 (32). P. 34-38.

11. Гуль В.Е., Шенфилд Л.3. Электропроводящие полимерные композиты. - М.: Химия, 1984. -226 с

12. Абраменко Е.А., Минакова Н.Н., Ушаков В.Я. Исследование свойств полиэтилена с наноразмерными наполнителями специальной обработкой изображений макроструктуры // Изве- практически не влияет на коэффициент вариации значений $\rho_{v t}$

Подбор материала матрицы и электропроводящего компонента позволяет формировать структуру с регулируемой степенью однородности (коэффициент вариации равномерности распределения фазовых составляющих на уровне 10 \% при действии повышенной температуры). Это способствует сокращению ресурсов, необходимых для производства электротехнических изделий с требуемыми электрофизическими характеристиками.

стия высших учебных заведений. Физика. - 2008. - Т. 51. № 7. - С. 39-42.

13. Минакова Н.Н., Сквирская И.И., Ушаков В.Я. Исследование природы нестабильности основных характеристик крупногабаритных полимерных резисторов // Электричество. - 2001. № 3. - C. 38-42

14. Special electrical conductivity of carbon black-filled two-phased thermoplastic vulcanizers / H. Tian, M. Tian, H., Zou Z. Dang, L. Zhang // Journal Applied Polymer Science. - 2010. - V. 117. Iss. 2. - P. 691-699.

15. Improvement of carbon black based polymer composite electrical conductivity with additions of MWCNT / N. Gorshkov, I. Ilinykh, D. Muratov, E. Kolesnikov, S. Anshin, I. Mazov, J.-P. Issi, D. Kusnezov // Composites Science and Technology. - 2016. V. 129. - P. 79-85.

16. Минакова Н.Н., Ушаков В.Я. Применение текстурного анализа к оценке работоспособности наполненных полимеров при повышенных температурах // Известия высших учебных заведений. Физика. - 2016. - Т. 58. - № 11. - С. 134-140.

17. Temperature coefficients of electrical conductivity and conduction mechanisms in butyl rubber-carbon black composites / M.A. Alzamil, K. Alfaramawi, S. Abboudy, L. Abulnasr // Journal of Electronic CK Materials. - 2018. - V. 47 (2). - P. 1665-1672.

18. Malas A., Das A. Influence of modified graphite flakes on the physical, thermo-mechanical and barrier properties of butyl rubber // Journal of Alloys and Compounds. - 2017. - V. 69 (9). - P. 38-46.

19. Enhancing the electrical conductivity of carbon black-filled immiscible polymer blends by tuning the morphology / Y. Pan, X. Liu, X. Hao, Z. Starý, D.W. Schubert // European Polymer Journal. - 2016. - V. 78. - P. 106-115.

20. Anaraki N.I., Poursalehi R. Shielding effectiveness of polymeric nanocomposites filled with iron/wüstite nanoparticles // Procedia Materials Science. - 2015. - № 11. - P. 700-705.

21. Topical issue on nanocomposites. preface: conductive polymer composites with segregated structures / Huan Pang, Ling $\mathrm{Xu}$, Ding-Xiang Yan, Zhong-Ming Li // Progress in Polymer Science. - 2014. - V. 39. - Iss. 11. - P. 1908-1933.

Поступила: 06.12.2019 г.

\section{Информация об авторах}

Минакова Н.H., доктор физико-математических наук, профессор, кафедра информационной безопасности Алтайского государственного университета.

Ушаков В.Я., заслуженный деятель науки и техники РФ, доктор технических наук, профессор отделения энергетики и электротехники Инженерной школы энергетики Национального исследовательского Томского политехнического университета. 
UDC 620.9:538.9

\title{
REGULATION OF REPRODUCTION OF POLYMER RESISTOR PARAMETERS AT INCREASED TEMPERATURES
}

\author{
Natalya N. Minakova1, \\ minakova@asu.ru \\ Vasily Ya. Ushakov², \\ vyush@tpu.ru \\ 1 Altai State University, \\ 61, Lenin avenue, Barnaul, 656049, Russia \\ 2 National Research Tomsk Polytechnic University, \\ 30, Lenin avenue, Tomsk, 634050, Russia.
}

The relevance of the work is caused by the fact that resistive polymer composite materials are actively used in various electrical devices. Diversified electrical devices are made of rubber filled with carbon black, having available feedstock and the possibility of industrial manufacturing technology. Their use is constrained by the complexity of the choice of components to provide the required electrophysical properties. The scatter of parameters associated with the multicomponent structure increases the cost of raw materials for the manufacture of finished products.

The main aim of the research is to assess the possibility of improving the reproducibility of properties in a series of electrical products for a specific field of operation due to prescription techniques.

The objects of the research are the instability (scatter) of the temperature coefficient of resistance, a change in the volume electric resistance when heated by direct or alternating electric current of various magnitude and duration. The studied composite materials differed in concentration, brand of the electrically conductive component, and the type of polymer.

Research methods: measurements of the electrophysical characteristics of resistive polymer composite materials, dispersion, regression, and correlation analyzes.

Results. The authors have determined the regularities of the behavior of the variation coefficient and the temperature coefficient of resistance with a change in the concentration of the conductive filler (soot) and the type of rubber when heated by direct and alternating current in the range of applied voltage up to $1000 \mathrm{~V}$. The authors developed recommendations on how to increase the uniformity of the structure of polymer composite materials designed to operate at elevated temperatures. The implementation of these recommendations helps to save the required resources (materials, energy, and time) due to the increase in reproducibility of the volume electrical resistivity when heating electrical products during operation.

\section{Key words:}

Resources saving, filled polymers, volume electric resistance, temperature coefficient of resistance, coefficient of variation, elevated temperature.

\section{References}

1. Brieu M., Diani J., Mignot C., Moriceau C. Stress softening of carbon black filled SBRs submitted to various large strain uniaxial tension cycles. Procedia Engineering, 2010, no. 1 (2), pp. 1291-1296.

2. Thomassina J.-M., Jérômea C., Pardoenb T., Baillyb C., Huynenb I., Detrembleura C. Polymer/carbon based composites as electromagnetic interference (EMI) shielding materials. Materials Science and Engineering, 2013, vol. 74, Iss. 7, pp. 211-232.

3. Zhang W., Dehghani-Sanij A.A., Blackburn. R.S. Carbon based conductive polymer composites. Journal Materials Science, 2007, vol. 42, pp. 3408-3418

4. Askadsky A.A., Khokhlov A.R. Vvedenie $v$ fiziko-khimiyu polimerov: monografiya [Introduction to the physical chemistry of polymers]. Moscow, Nauchny mir Publ., 2009. 384 p

5. Lipatov Yu.S. Fiziko-khimicheskie osnovy napolneniya polimerov [Physical and chemical bases of polymer filling]. Moscow, Khimiya Publ., 1977. 260 p.

6. Alzamil M.A., Alfaramawi K., Abboudy S., Abulnasr L. Electrical conduction hysteresis in carbon black-filled butyl rubber compounds. International Journal of Modern Physics, 2018, no. 32 (09), pp. $1-10$

7. Berki P., Göbl R., Karger-Kocsis J. Structure and properties of styrene-butadiene rubber (SBR) with pyrolytic and industrial carbon black. Polymer Testing, 2017, vol. 61, pp. 404-415.

8. Szadkowski B., Marzec A., Zaborski M. Effect of different carbon fillers on the properties of nitrile rubber composites. Composite Interfaces, 2019, vol. 26 (8), pp. 729-750.
9. Janke W. Computer simulation studies of polymer adsorption and aggregation - from flexible to stiff. Physics Procedia, 2015, no. 68 pp. 69-79.

10. Liu X., Pan Y., Hao X., Dai K., Schubert D.W. The role of conductive pathways in the conductivity and rheological behavior of poly (methyl methacrylate)-graphite composites. Journal of Applied Polymer Science, 2016, no. 133 (32), pp. 34-38.

11. Gul V.E., Shenfild L.Z. Elektroprovodyashchie polimernye kompozity [Electrically conducting polymer compositions]. Moscow, Khimiya Publ., 1984. 226 p.

12. Abramenko E.A., Minakova N.N., Ushakov V.Ya. Investigation of the properties of polyethylene with nanodimensional fillers by special processing of macrostructure images. Russian Physics Journal, 2008, vol. 51 (7), pp. 701-705. In Rus.

13. Minakova N.N., Skvirskaya I.I., Ushakov V.Ya. Issledovanie prirody nestabilnosti osnovnykh kharakteristik krupnogabaritnykh polimernykh rezistorov [Investigation of the instability nature of main characteristics or large size polymer resistors]. Elektrichestvo, 2001, no. 3, pp. 38-42.

14. Tian H., Tian M., Zou H., Dang Z., Zhang L. Special electrical conductivity of carbon black-filled two-phased thermoplastic vulcanizates. Journal Applied Polymer Science, 2010, vol. 117, Iss. 2, pp. 691-699.

15. Gorshkov N., Ilinykh I., Muratov D., Kolesnikov E., Anshin S., Mazov I., Issi J.-P., Kusnezov D. Improvement of carbon black based polymer composite electrical conductivity with additions of MWCNT. Composites Science and Technology, 6 June 2016, vol. 129, pp. 79-85. 
16. Minakova N.N., Ushakov V.Ya. Application of texture analysis to assess the operability of filled polymers at high temperatures. Russian Physics Journal, 2016, vol. 58, no. 11, pp. 1627-1634. In Rus.

17. Alzamil M.A., Alfaramawi K., Abboudy S., Abulnasr L. Temperature coefficients of electrical conductivity and conduction mechanisms in butyl rubber-carbon black composites. Journal of Electronic CK Materials, 2018, vol. 47 (2), pp. 1665-1672.

18. Malas A., Das A. Influence of modified graphite flakes on the physical, thermo-mechanical and barrier properties of butyl rubber. Journal of Alloys and Compounds, 2017, vol. 69 (9), pp. 38-46.

19. Pan Y., Liu X., Hao X., Starý Z., Schubert D.W. Enhancing the electrical conductivity of carbon black-filled immiscible polymer

\section{Information about the authors}

Natalya N. Minakova, Dr. Sc., professor, Altai State University.

Vasily Ya. Ushakov, Honored Scientist and Engineer of the RF, Dr. Sc., professor, National Research Tomsk Polytechnic University. blends by tuning the morphology. European Polymer Journal, 2016, vol. 78, pp. 106-115.

20. Anaraki N.I., Poursalehi R. Shielding effectiveness of polymeric nanocomposites filled with iron/wüstite nanoparticles. Procedia Materials Science, 2015, no. 11, pp. 700-705.

21. Huan Pang, Ling Xu, Ding-Xiang Yan, Zhong-Ming Li. Topical issue on nanocomposites. Preface: conductive polymer composites with segregated structures. Progress in Polymer Science, 2014, vol. 39, Iss. 11, pp. 1908-1933.

Received: 6 December 2019. 